\title{
Apoyando la adquisición de competencias científicas a través de Analíticas del Aprendizaje
}

\author{
Daniel J. Salas ${ }^{1}$, Silvia M. Baldiris ${ }^{2}$, Ramon Fabregat ${ }^{2}$, Sabine Graf ${ }^{3}$ \\ ${ }^{1}$ Grupo Socrates, Universidad de Córdoba, Montería, Colombia \\ ${ }^{2}$ University of Girona, Institute of Informatics and Applications, Girona, Spain \\ ${ }^{3}$ Athabasca University, School of Computing and Information Systems, Edmonton, \\ Canada
}

danielsalas@correo.unicordoba.edu.co,baldiris@eia.udg.edu,ramon.fabre gateudg.edu, sabinegeathabascau.ca

Resumen. El propósito de este trabajo es plantear analíticas de aprendizaje para apoyar la adquisición de competencias científicas en estudiantes universitarios que inician procesos de investigación a través de la reflexión sobre su proceso de aprendizaje, para de esta manera ayudarlos a comprender las posibles limitaciones de comprensión de los conceptos, fases, etapas y aspectos de relevancia que son de interés en las tareas llevadas a cabo para la generación de conocimiento cientifíco.

Abstract. The purpose of this paper is to learning analytics to support the acquisition of scientific skills in academic students who start research processes through reflection on their learning process, and in this way help them understand the potential limitations of understanding concepts, phases, stages and aspects of relevance that are of interest in the work carried out for the generation of scientific knowledge

\section{Introducción}

La formación investigativa cada día es mas relevante y de interés en las instituciones educativas en todos los niveles y grados de formación, esta afirmación es soportada en diversos estudios, entre ellos el de la UNESCO [UNESCO 2006], en el que se exponen varias razones de suma importancia como: la contribución a la formación del pensamiento lógico a través de la resolución de problemas, el mejoramiento de la calidad de vida, la preparación para la inserción en el mundo científico, la explicación de las realidades del mundo en su contexto, entre otros.

La OCDE ha venido impulsando una política relacionada con las competencias científicas, que ha quedado explicita en las pruebas PISA [OCDE 2006]. La OCDE entiende las competencias cientificas como "el conocimiento científico y el uso que se hace de ese conocimiento para identificar cuestiones, adquirir nuevos conocimientos, explicar fenómenos científicos y extraer conclusiones basadas en pruebas". 
Los procesos de investigación generalmente incluyen un amplio conjunto de tipos de métodos y técnicas de investigación que podrían ser utilizados y aplicados por los investigadores principiantes. Sin embargo, cuando ellos se enfrentan a la necesidad de definir un estudio de investigación, se encuentran con muchos problemas como: la percepción de los jóvenes investigadores sobre la orientación de los asesores, la falta de claridad conceptual sobre los métodos y técnicas de investigación, la dificultad en la revisión de literatura científica, la poca habilidad en los diseños de investigación, acentuados problemas en el análisis, interpretación de los datos y en los escenarios de evaluación [Reeves 2000].

La problemática anterior requiere de nuevos enfoques y estrategias innovadoras que incorporen elementos que faciliten el seguimiento y apoyen de manera significativa la adquisición de competencias científicas. En este sentido, se considera que las analíticas de aprendizaje pueden constituirse en una herramienta conceptual y tecnológica útil. En este artículo se introduce una propuesta de analíticas de aprendizaje para promover y fortalecer la reflexión de los investigadores principiantes sobre su avance en la adquisición de competencias científicas y en la comprensión crítica de sus nuevos procesos de investigación.

El articulo está estructurado de la siguiente manera. :En la primera sección está la introducción. En la segunda sección se presenta el estado del arte y en la tercera sección la propuesta de analíticas para las competencias científicas. En la cuarta sección propone un escenario de validación y finalmente en la quinta y sexta sección se incluyen las conclusiones y el trabajo futuro.

\section{Estado del Arte}

\subsection{Competencias Científicas}

La revisión de literatura sobre competencias científicas destaca que los referentes más relevantes son las planteamientos de la UNESCO con la alfabetización científica [UNESCO 2006], las políticas de la OCDE implementadas mediante las pruebas PISA que tienen incidencia en jovenes de 15 a 18 años [OCDE 2006] y el proyecto TUNING, que en sus versiones para Europa y América Latina [TUNNING,2000,2004] ha definido competencias cientificas para estudiantes de pregrado. También es sobresaliente la experiencia de Australia [ACARA,2012] a través de las estrategias desarrolladas por el Ministerio de Educación de ese país con resultados alentadores para el fortalecimiento de los procesos de investigación. En Estados Unidos, a través de la [NPA,2005], se ha definido un conjunto de competencias científicas de relevancia en el orden global para doctorado y posdoctorado. En el caso colombiano, el gobierno a través del Ministerio de Educación Nacional [MEN,2005] ha definido un conjunto de compentencias científicas para todos los niveles de formación (educación básica, secundaria y educación superior).

En este trabajo se propondrán analíticas de aprendizaje para apoyar la adquisición de competencias científicas de estudiantes de pregrado, en particular para el caso colombiano,cuya evaluación de dichas competencias según lo planteado por [Brown, 2013] y [Montenegro,2009], se requiere la integración de enfoques cualitativos y cuantitativos, también expuesto por [Bujan, 2013] y [Muñoz, 2001] 
CBIE-LACLO 2015

Anais dos Workshops do IV Congresso Brasileiro de Informática na Educação (CBIE 2015)

\subsection{Analíticas de Aprendizaje.}

Las Analíticas de Aprendizaje, como área de investigación emergente, tienen sus fundamentos en los conceptos de datos masivos (Big Data en inglés), la inteligencia de negocios (Businness Intelligent (BI) en inglés) y la minería de datos (Data Mining en inglés). Esta última ha tenido como extensión el término Minería de Datos Educativa (Educational Data Mining en inglés) [Romero y Ventura 2007].

La aparición del concepto de Analíticas de Aprendizaje ha sido motivado por el uso masivo de los Virtual Learning Enviroment (VLE), los Adaptative Hypermedia System (SHA) y los Personal Learning Enviroment (PLE), así como por los avances de la computación y del aprendizaje ubícuo que generan grandes cantidades de datos e información por la interacción de los estudiantes y de los profesores con este tipo de sistemas.

Existen varias definiciones ampliamente conocidas sobre las análiticas de aprendizaje, por ejemplo [Siemmens 2010], [Duval 2011], [Elias 2011] y [UNESCO 2012], sin embargo, la definición comúnmente aceptada es la planteada por la conferencia [LAK 2011] que define el término como "La medición, recopilación, análisis y presentación de datos sobre los aprendices y sus contextos, a efectos de entender y optimizar el aprendizaje y el entorno en que se produce".

Una primera aproximación de clasificación de analíticas desde la perspectiva del SLA (Social Learning Analitics) fue planteada por [Fergunson y Buckingham 2011) y [Buckingham y Fergunson, 2012]. En estos estudios se definen cinco categorias de las analíticas divididas en dos grupos. El primero conformado por el análisis de redes sociales y el análisis del discurso. El segundo conformado por el análisis del contenido, el análisis de la motivación y el análisis del contexto.

\section{Proceso de adquisición de competencias científicas: el caso Colombiano}

Las unidades didácticas del proceso de formación en competencias cientifícas que abordará el estudiante tendran la siguiente estructura.

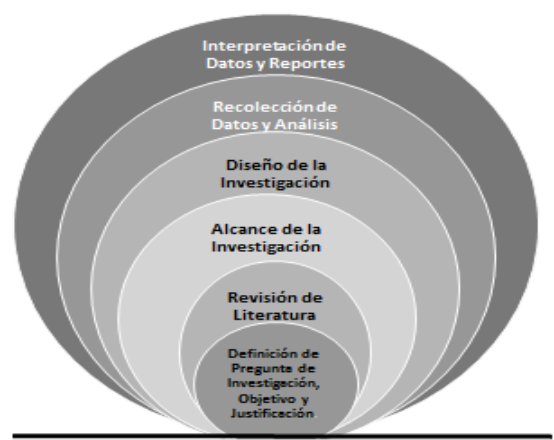

Figura 1. Esquema para el proceso formativo 
El gobierno colombiano ha definido un conjunto de competencias científicas que deben ser desarrolladas por los estudiantes de pregrado en cada una de las seis fases del proceso científico, que hemos definido en este trabajo

A continuación se relacionan las analíticas asociadas a cada una de las fases del proceso para apoyar la adquisición de competencias:

F1 - Relativas a la calidad en el proceso de observación.

- $\mathrm{C} 1$ - Habilidad para observar, reconocer y detectar particularidades.

- C2 - Habilidad para realizar observaciones y emplearlas en la formulación de preguntas y problemas de investigación.

- Asociaciones de conceptos realizadas.

F2 - Relativas a la calidad en la búsqueda y gestión de información científica.

- C3 - Capacidad para identificar conocimiento científico, es decir, qué es lógico, verificable, reproducible, sistemático, universal, comunicable y dinámico.

F3 - Relativas a la calidad en las preguntas de investigación, en el planteamiento del problema de investigación, en los objetivos, en la justificación y en el alcance de la investigación.

- C4 - Habilidad para construir preguntas de investigación a partir de un conjunto de observaciones, datos y referentes teóricos.

F4 - Relativas a la calidad en el diseño de la investigación.

- C5 - Habilidad para plantear diseños metodológicos apropiados de acuerdo con los objetivos de investigación.

F5 - Relativas a la calidad en la recoleción y en el analísis de datos.

- C6 - Habilidad para utilizar procedimientos y técnicas apropiados para lograr confiabilidad en la información.

F6 - Relativas a la calidad en la interpretación de datos y en los informes científicos.

- C7 - Habilidad para interpretar resultados y representarlos adecuadamente.

- C8 - Habilidad para plantear conclusiones pertinentes a los resultados de investigación.

\section{Analíticas de aprendizaje para el proceso de adquisición de competencias cientficas}

Con base en las competencias científicas mencionadas previamente que agrupan las capacidades específicas que deben desarrollar los estudiantes universitarios de pregrado en Colombia, se han propuesto las siguientes analíticas de aprendizaje (ver tabla 1), igualemente se definen las metrícas y herramientas de medición, desde una perspectiva amplia que considera la necesidad de contar instrumentos que involucren los aspectos cualitativos y cuantitativos, con el fin de tener una aproximación autentica y eficiente en la evaluación de competencias cientificas. 
CBIE-LACLO 2015

Anais dos Workshops do IV Congresso Brasileiro de Informática na Educação (CBIE 2015)

Tabla 1. Analíticas para competencias científicas.

\begin{tabular}{|c|c|c|}
\hline Analiticas por fases & Metrícas & $\begin{array}{c}\text { Herramientas de } \\
\text { Medición }\end{array}$ \\
\hline $\begin{array}{l}\text { Relativas a la calidad en el } \\
\text { proceso de observación. } \\
\text { (Fase 1) }\end{array}$ & $\begin{array}{l}\text { - Grado de comprensión de } \\
\text { conceptos. } \\
\text { - } \text { Interpretaciones conceptuales. } \\
\text { - } \text { Realizadaciones conceptuales. } \\
\text { - Clasificaciones conceptuales } \\
\text { realizadas. } \\
\text { - Categorizaciones conceptuales } \\
\text { realizadas. } \\
\text { - Agrupaciones conceptuales } \\
\text { realizadas. } \\
\text { Asociaciones de conceptos } \\
\text { realizadas. }\end{array}$ & $\begin{array}{l}\text { Fichas de } \\
\text { observación } \\
\text { sistemática. }\end{array}$ \\
\hline $\begin{array}{l}\text { Relativas a la calidad en la } \\
\text { búsqueda y gestión de } \\
\text { información científica. } \\
\text { (Fase } 2 \text { ) }\end{array}$ & $\begin{array}{l}\text { - Fuentes consultadas. } \\
\text { - Fuentes especializadas. } \\
\text { - } \text { en español. } \\
\text { - } \text { idiontes consultadas. } \\
\text { - Técnicas para la selección de } \\
\text { - fuentes de datos. } \\
\text { - } \text { Revistas especializadas en la } \\
\text { - Capitica consultadas. } \\
\text { - Tesis de maestría consultadas. } \\
\text { - Tesis de doctorado consultadas. } \\
\text { Numéro de bases de datos } \\
\text { consultadas. }\end{array}$ & 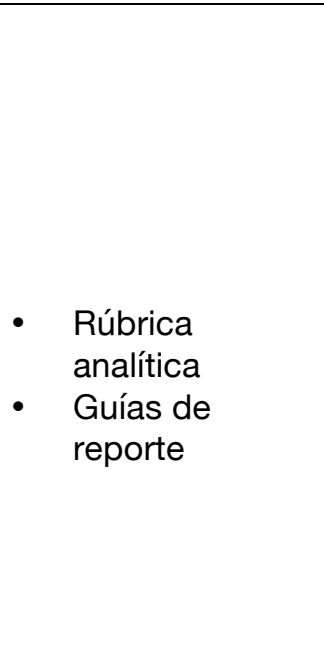 \\
\hline $\begin{array}{l}\text { Relativas a la calidad en las } \\
\text { preguntas de investigación, en } \\
\text { el planteamiento del problema } \\
\text { de investigación, en los } \\
\text { objetivos, en la justificación y } \\
\text { en el alcance de la } \\
\text { investigación. (Fase 3) }\end{array}$ & $\begin{array}{ll}\text { - } & \text { Preguntas formuladas. } \\
\text { - } & \text { Preguntas abiertas formuladas. } \\
\text { - } & \text { Preguntas cerradas formuladas. } \\
& \text { formuladas. } \\
\text { - } & \text { Preguntas irrelevantes. } \\
\text { - } & \text { Preguntas viables de ser } \\
\text { investigadas. } \\
\text { - Preguntas no viables de ser } \\
\text { investigadas. } \\
\text { - Grado de complejidad del } \\
\text { problema. } \\
\text { - } \text { investerencia del problema de } \\
\text { Objetivos medibles y } \\
\text { verificables. } \\
\text { - } \text { Objetivos no medibles. } \\
\text { investigación. } \\
\text { - Grado de pertinencia } \\
\text { tecnológica de la investigación. }\end{array}$ & $\begin{array}{l}\text { - } \text { Portafolio } \\
\text { - Guías } \\
\text { problémicas de } \\
\text { diseño } \\
\text { - Guías } \\
\text { problémicas de } \\
\text { no diseño }\end{array}$ \\
\hline
\end{tabular}


CBIE-LACLO 2015

Anais dos Workshops do IV Congresso Brasileiro de Informática na Educação (CBIE 2015)

\begin{tabular}{|c|c|c|}
\hline Analiticas por fases & Metrícas & $\begin{array}{l}\text { Herramientas de } \\
\text { Medición }\end{array}$ \\
\hline & $\begin{array}{l}\text { - Grado de relevancia científica de } \\
\text { la investigación. }\end{array}$ & \\
\hline $\begin{array}{l}\text { Relativas a la calidad en el } \\
\text { diseño de la investigación. } \\
\text { (Fase 4) }\end{array}$ & $\begin{array}{l}\text { - Número de métodos cualitativos } \\
\text { de investigación consultados y } \\
\text { apropiados. } \\
\text { - Número de métodos } \\
\text { cuantitativos de investigación } \\
\text { consultados y apropiados. } \\
\text { - Técnicas cuantitativas de } \\
\text { investigación consultadas y } \\
\text { apropiadas. } \\
\text { - Técnicas cualitativas de } \\
\text { investigación consultadas y } \\
\text { apropiadas. } \\
\text { Pertinencia del método de } \\
\text { investigación utilizado en } \\
\text { investigaciones realizadas. }\end{array}$ & $\begin{array}{l}\text { - } \text { Portafolio } \\
\text { - Rúbrica } \\
\text { comprensiva }\end{array}$ \\
\hline $\begin{array}{l}\text { Relativas a la calidad en la } \\
\text { recoleción y en el analísis de } \\
\text { datos. (Fase 5) }\end{array}$ & $\begin{array}{l}\text { - Técnicas para la recolección de } \\
\text { datos apropiadas. } \\
\text { - Técnicas para recolección de } \\
\text { datos seleccionadas } \\
\text { adecuadamente. } \\
\text { - Instrumentos de medición } \\
\text { apropiados. } \\
\text { - Instrumentos de medición } \\
\text { seleccionados adecuadamente. } \\
\text { - Confiabilidad y validez de los } \\
\text { instrumentos de medición } \\
\text { construidos. } \\
\text { - Técnicas estadísticas para el } \\
\text { análisis de datos apropiadas. } \\
\text { - Técnicas estadísticas para el } \\
\text { análisis de datos seleccionadas } \\
\text { adecuadamente. } \\
\text { - Software analizados para } \\
\text { analisis de datos. } \\
\text { Uso adecuado de software para } \\
\text { analisis de datos. }\end{array}$ & $\begin{array}{ll}\text { - } & \text { Cuestionarios } \\
\text { - } & \text { Guía y análisis } \\
\text { de ejercicios } \\
\text { - } & \text { Pruebas de } \\
& \text { rendimiento }\end{array}$ \\
\hline $\begin{array}{l}\text { Relativas a la calidad en la } \\
\text { interpretación de datos y en } \\
\text { los informes científicos. (Fase } \\
6 \text { ) }\end{array}$ & $\begin{array}{l}\text { - Técnicas para interpretación de } \\
\text { datos consultadas. } \\
\text { - Herramientas para la } \\
\text { interpretación de datos } \\
\text { consultadas. } \\
\text { - Técnicas para interpretación de } \\
\text { datos usadas adecuadamente. } \\
\text { - Herramientas para la } \\
\text { interpretación de datos usadas } \\
\text { adecuadamente. } \\
\text { - Grado de coherencia y } \\
\text { consistencia de la conclusiones. } \\
\text { Herramientas consultadas y } \\
\text { apropiadas para la edición de } \\
\text { información científicas. }\end{array}$ & 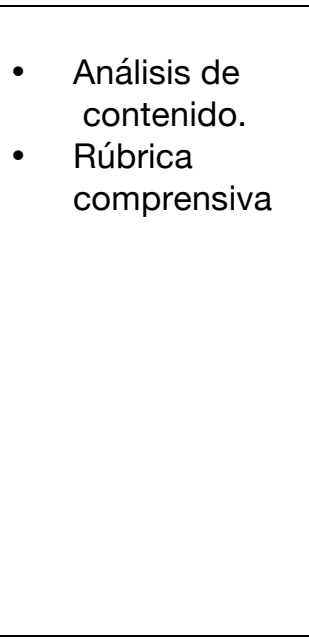 \\
\hline
\end{tabular}


CBIE-LACLO 2015

Anais dos Workshops do IV Congresso Brasileiro de Informática na Educação (CBIE 2015)

\begin{tabular}{|l|l|l|}
\hline Analiticas por fases & \multicolumn{1}{|c|}{ Metrícas } & $\begin{array}{c}\text { Herramientas de } \\
\text { Medición }\end{array}$ \\
\hline & $\bullet \begin{array}{l}\text { Herramientas seleccionadas } \\
\text { para la edición científica. } \\
\text { Herramientas consultadas para } \\
\text { la presentación de reportes de } \\
\text { información científica. }\end{array}$ & \\
\hline
\end{tabular}

\section{Descripción del escenario de validación}

Se propone el desarrollo de un curso virtual en competencias científicas articulado a una red social de aprendizaje y un visualizador inteligente de datos que mostraría el comportamiento de la adquisión de competencias con base en las metricas y analiticas definidas para los estudiantes de semilleros de investigación de los programas de ingenierias en la Universidad de Córdoba. Un esquema de implementación se presenta en el siguiente gráfico.

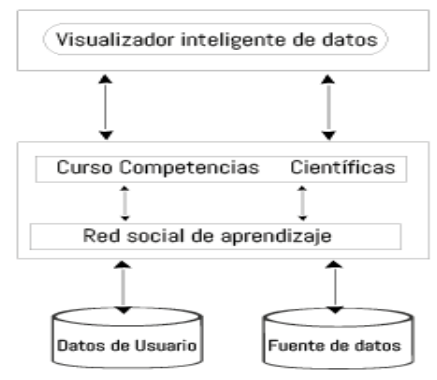

Figura 2. Esquema de implementación de competencias científicas

\section{Conclusiones}

En este trabajo se ha presentado una aproximación a un planteamiento de analíticas de aprendizaje para apoyar la adquisición de comptencias científicas con un esquema de metricas y herramientas de medición para aplicar a jovenes universitarios mediante una propuesta de implementación con una red social de aprendizaje articulada con un curso virtual.

\section{Referencias.}

ACARA, (2012) National Assessment Program - Science Literacy Year 6 Report.

Buckingham,S, et al (2012) Social Learning Analytics, Educational Technology \& Society, 15 (3), 3-26.

Bujan Vidales, K; Rekalde Rodríguez, I; Aramendi Jáuregui, P. (2011). La evaluación de competencias en la educación superior. las rúbricas como instrumento de evaluación. Bogotá: ediciones de la U.

Brown, S; Pickford, R. (2013). Evaluación de habilidades y competencias en Educación superior. España: ediciones Narcea. 
Duval, E and Verbert, K. (2011) Learning Analytics

Elias, T (2011) Learning Analytics: Definitions, Processes and potential,

Fergunson, R. and Buckingman, S (2012). The state of Learning Analytics in 2012 : A review and future challenges, Technical report KMI 12-0

Fergunson, R. et al (2012) Social Learning Analytics: Five Approaches

Montenegro Aldana, I. (2009). Cómo evaluar el aprendizaje escolar. Bogotá: editorial magisterio.

Ministerio de Educación Nacional,(2005) Competencias Científicas.

Muñoz Giraldo, J; Quintero Corzo, J; Munévar Molina, R. (2001). Cómo desarrollar competencias investigativas en educación. Bogotá: editorial aula abierta magisterio.

National Postdoctoral Association(2007), The NPA Postdoctoral Core Competencies

LAK (2011), 1st International Conference on Learning Analytics and Knowled

OCDE, PISA (2006), Marco de la evaluación. Conocimiento y habilidades en ciencias, matemáticas y lectura.

Romero, C. and Ventura, S. (2007). Educational Data Mining: A Survey from 1995 to 2005. Expert Systems with Applications 33, 125-146.

Reeves, T. (2000) Enhancing the Worth of Instructional Technology Research through "Design Experiments" and Other Development Research Strategies.

Siemens, G. 2010. What is learning analytics?. http://www.elearnspace.org/blog/2010/08/ 25/what-are learning analytics Retrieved 11 Octuber 2011.

Tuning Educational Structures in Europe (2000).

Tuning (2004), Reflexiones y perspectivas de la educación superior en América Latina.

UNESCO. (2005) ¿ Cómo promover el interés por la cultura cientifica? Una propuesta didáctica fundamentada para la educación cientifica de jovenes de 15 a 18 años.

UNESCO, (2012) Policy Brief, Learning Analytics 